\title{
Atuação do enfermeiro no protocolo de cirurgia segura: uma intervenção no pronto socorro
}

\author{
Nurse's role in the safe surgery protocol: an intervention in the emergency room
}

El papel de la enfermera en el protocolo de cirugía segura: una intervención en urgencias

Tainara Rodrigues de Araujo1*, Joyce Lora Gomes da Silva ${ }^{1}$, Eliene Rodrigues dos Passos ${ }^{1}$, Cristiane Oliveira Secundo ${ }^{1}$.

\section{RESUMO}

Objetivo: Construir uma proposta de intervenção a partir da análise de fatores que interem na atuação do enfermeiro no processo de cirurgia segura, promovendo assim a transformação da realidade, no pronto socorro em Rondônia. Métodos: Trata-se de uma pesquisa com abordagem qualitativa e de intervenção, utilizado como base a teoria da problematização do arco de Charles Maguerez, sendo realizada em um hospital de pronto atendimento em Rondônia. Resultados: Elencados três núcleos temáticos: Conhecimento do Enfermeiro Sobre o Protocolo de Cirurgia Segura; A Importância do Enfermeiro Frente ao Processo de Cirurgia Segura; Dificuldades para Realização do Checklist no Serviço. Como intervenção foi realizado rodas de conversa com os profissionais enfermeiros, promovendo assim uma discussão crítica e reflexiva sobre o processo de trabalho envolvendo toda a caminhada na cirurgia segura para o paciente. Conclusão: Propiciou aperfeiçoamento na qualidade de assistência visando a segurança do paciente, do mesmo modo a consolidação das relações entre a equipe multiprofissional.

Palavras-chave: Segurança do paciente, Protocolo, Assistência de enfermagem.

\begin{abstract}
Objective: To build an intervention proposal based on the analysis of factors that influence the role of nurses in the safe surgery process, thus promoting the transformation of reality in the emergency room in Rondônia. Methods: This is a research with a qualitative and intervention approach, based on Charles Maguerez's arch problematization theory, being carried out in an emergency care hospital in Rondônia. Results: Three thematic cores were listed: Nurse's Knowledge about the Safe Surgery Protocol; The Importance of the Nurse in the Safe Surgery Process; Difficulties in Carrying Out the Checklist in the Service. As an intervention, conversation circles were held with the nursing professionals, thus promoting a critical and reflective discussion about the work process involving the entire journey in safe surgery for the patient. Conclusion: Provided improvement in the quality of care aimed at patient safety, as well as the consolidation of the relationship between the multidisciplinary team.
\end{abstract}

Keywords: Patient safety, Protocol, Nursing care.

\section{RESUMEN}

Objetivo: Construir una propuesta de intervención basada en el análisis de los factores que influyen en el papel del enfermero en el proceso de cirugía segura, promoviendo así la transformación de la realidad en el servicio de urgencias de Rondônia. Métodos: Se trata de una investigación con enfoque cualitativo e intervencionista, basada en la teoría de la problematización del arco de Charles Maguerez, que se lleva a cabo en un hospital de urgencias de Rondônia. Resultados: Se enumeraron tres ejes temáticos: Conocimiento de la enfermera sobre el Protocolo de Cirugía Segura; La importancia del enfermero en el proceso quirúrgico seguro; Dificultades para la realización de la lista de verificación en el servicio. Como intervención, se realizaron círculos de conversación con los profesionales de enfermería, promoviendo así una discusión crítica y reflexiva sobre el proceso de trabajo que involucra todo el recorrido en cirugía segura para el paciente. Conclusión: Proporcionó la mejora en la calidad de la atención orientada a la seguridad del paciente, así como la consolidación de la relación entre el equipo multidisciplinario.

Palabras clave: Seguridad del paciente, Protocolo, Atención de enfermería.

${ }^{1}$ Faculdade Interamericana de Porto Velho - UNIRON, Porto Velho - RO.

*E-mail: tainara_enfer@hotmail.com

SUBMETIDO EM: 6/2021 


\section{INTRODUÇÃO}

Em 2004 a Organização Mundial da Saúde (OMS) criou a Aliança Mundial para a Segurança do Paciente, como propósito de despertar a consciência profissional e o envolvimento político para a melhoria da segurança na assistência à saúde. Nesse mesmo contexto, foram formulados os Desafios Globais para a Segurança do Paciente tendo como primeiro deles as infecções relacionadas à assistência à saúde. Em 2007/2008, o Segundo Desafio Global com a campanha intitulada Cirurgia Seguras Salvam Vidas, que teve como enfoque a segurança cirúrgica, com proposta para a melhoria de processos assistências e redução de mortes e complicação no decorrer das cirurgias (SILVA HR, et al., 2020).

A segurança do paciente visa evitar, prevenir ou minimizar eventos adversos ou lesões durante 0 atendimento médico-hospitalar e domiciliar (SILVA ACA, et al., 2016). Atualmente os eventos adversos com maior incidência, é a cirurgia em local errado, principalmente na área da ortopedia. O evento adverso também pode acontecer pela falha durante a identificação do paciente, ao ser referido pelo número do leito, ou pacientes com o mesmo nome (CRIADO JVN, et al., 2017).

De acordo com Barbosa GA, et al. (2018), a Lista de Verificação de Cirurgia Segura (LVCS), é composta por três etapas, descritas como: Identificação (Sign In), Confirmação (TimeOut) e o Registro (SignOut). O primeiro momento acontece antes da indução anestésica, o segundo momento ocorre com a incisão cirúrgica e o terceiro momento corresponde ao final do procedimento realiza-se o registro.

De acordo com Borges F, et al. (2016), os enfermeiros cumprem papel importante na fase pré, trans e pós-operatória, sendo relevante na transmissão de confiança e segurança ao paciente. Contudo vale ressaltar que, a prática de enfermagem exige que os profissionais estejam preparados quanto ao conhecimento técnico e interação de todas as etapas do processo cirúrgico que implicam na segurança do paciente, bem como realizar um cuidado humanizado favorecendo a cirurgia segura.

Como contribuição atribui o esclarecimento da importância do papel do profissional enfermeiro no protocolo de cirurgia segura, sugestão de novos estudos e também discussões para as melhores práticas. Instigou-se a questão norteadora desta pesquisa: Como se dá a atuação do enfermeiro no processo de Cirurgia Segura? Como objetivo principal construir uma proposta de intervenção a partir da análise de fatores que interfere na atuação do enfermeiro no processo de cirurgia segura, promovendo assim a transformação da realidade.

\section{MÉTODOS}

Trata-se de uma pesquisa com abordagem qualitativa e de intervenção, utilizado como base a teoria da problematização do arco de Charles Maguerez, sendo realizada em um hospital de pronto atendimento em Rondônia. Para constituição da amostra os critérios de inclusão: enfermeiros que atuam no pré, intra e pósoperatório, nos setores de sala vermelha, centro cirúrgico e enfermarias. Foram convidados voluntariamente 18 enfermeiros(as) que estavam disponíveis em seus devidos setores, que concordaram em participar do estudo por meio da assinatura do Termo de Consentimento Livre e Esclarecido (TCLE).

A análise dos dados foi realizada concomitante ao desenvolvimento da primeira etapa da metodologia do Arco de Maguerez que é a observação da realidade. Nesta foi realizada a aplicação do questionário aos enfermeiros, com perguntas abertas o que caracterizou uma pesquisa com abordagem qualitativa, sucedendo a análise de dados baseado em Minayo MCS (2013) e a comparação desses com a literatura.

Através das respostas obtidas, as pesquisadoras dividiram em categorias com base nas questões convergentes e comparados com achados da literatura. Os participantes da pesquisa foram identificados pelas siglas E1, E2, e assim por diante, de modo a preservar o anonimato dos participantes.

Este trabalho sucedeu-se com base preceito da Resolução n. ㄴ 466, de 12 de dezembro de 2012 do Conselho Nacional de Saúde/Ministério da Saúde, com número do parecer: 3.668.904, Certificado de Apresentação de Apreciação Ética (CAAE), o número: 20832119.9.0000.8028, com data: 29 de outubro de 2019 com CEP da União Educacional do Norte LTDA (UNINORTE). 


\section{RESULTADOS E DISCUSSÃO}

\section{Primeira etapa: observação da realidade}

Iniciou-se somente após a aprovação do CEP, ocorreu o primeiro contato dos pesquisadores com os profissionais enfermeiros, com apresentação da pesquisa e seu objetivo, realizado a entrega do TCLE e após aceite da participação da pesquisa, ocorreu a assinatura do referido termo.

O questionário elaborado pelas pesquisadoras, continha duas subseções: caracterização dos enfermeiros com seis perguntas abertas. A coleta de dados com o uso do questionário nesta fase de observação da realidade, serviu de subsídio para obter a etapa diagnóstica da pesquisa com a definição do problema identificado no momento em que os dados foram coletados pelas pesquisadoras.

\section{Segunda etapa: definição dos pontos-chave}

Ocorreu a análise dos dados coletados, mediante a aplicação do questionário, realizando assim uma leitura para caracterizar os pontos chave com a reflexão dos enfermeiros sobre a importância do protocolo de cirurgia segura. A partir desta reflexão, iniciou-se o momento de buscado que ia ser estudado, trazendo assim os aspectos que precisavam ser conhecidos sobre o problema na aplicação da Lista de Verificação de Cirurgia Segura (LVCS) e desta forma intervir com a ação necessária. Nesse contexto foram identificados os pontos chave descritos no quadro abaixo.

Quadro 2 - Pontos-chave encontrados.

\begin{tabular}{|c|l|}
\hline Sessão & \multicolumn{1}{|c|}{ Ponto Chave } \\
\hline 1 & Preenchimento incompleto e inadequado das etapas do checklist; \\
\hline 2 & $\begin{array}{l}\text { A atribuição do preenchimento do checklist a somente um membro da equipe que } \\
\text { deveria estar presente em todas as salas cirúrgicas; }\end{array}$ \\
\hline 3 & $\begin{array}{l}\text { Resistência de alguns integrantes da equipe multiprofissional em responder } \\
\text { verbalmente as etapas do checklist; }\end{array}$ \\
\hline 4 & Opreenchimento inadequado das etapas do checklist; \\
\hline 5 & $\begin{array}{l}\text { Não adesão completa ao protocolo cirurgia segura que culminava em tensão e } \\
\text { desarmonia na relação entre equipe multiprofissional. }\end{array}$ \\
\hline
\end{tabular}

Fonte: AraujoTR, et al., 2021.

\section{Terceira etapa: teorização}

A teorização sucedeu por meio da investigação em artigos científicos em fontes de pesquisa com a literatura pertinente. Ressalta-se, que a pesquisa bibliográfica realizada foi um importante elemento que serviu para subsidiar o problema, os pontos chaves, as hipóteses e as diretrizes que orientaram a pesquisa. Sendo elencados três núcleos temáticos: Conhecimento do Enfermeiro Sobre o Protocolo de Cirurgia Segura; A Importância do Enfermeiro Frente ao Processo de Cirurgia Segura; Dificuldades para Realização do Checklist no Serviço.

\section{Conhecimento do enfermeiro sobre o protocolo de cirurgia segura}

Dentro dos achados das falas o conhecimento dos participantes baseava-se em ser um protocolo usado para garantir um processo cirúrgico eficaz não somente para o paciente mais também para toda a equipe envolvida na assistência. Segundo Lopes MCR, et al. (2019) o protocolo de cirurgia segura garante uma assistência adequada ao paciente e o desenvolvimento de um processo assistencial seguro para toda a equipe de enfermagem, ajudando na prevenção de falhas e na minimização dos eventos causados pela assistência inadequada.

De acordo com o que foi colocado pelos enfermeiros eles demonstraram ter conhecimento sobre o protocolo de cirurgia segura:

"Procedimento adotado para se garantir um processo cirúrgico adequado através do histórico do paciente e dos envolvidos" (E1). 
"Protocolo para estabelecer segurança no processo de cirurgia, em todas as suas fases. Dando ao cliente seguridade de vida e ao profissional, seguridade a suas ações" (E16).

Diante disso, notou-se que o conhecimento dos participantes firma-se na vertente de que, o protocolo é um processo que se for realizado de forma fidedigna, acarretará seguridade para todos os envolvidos, além de proporcionar a efetividade do desenvolvimento da assistência. Oliveira MCB, et al. (2018) nos traz que, quando o cheklist é aplicado de forma correta a equipe de saúde fica mais segura em desenvolver suas ações dentro do Centro cirúrgico (CC) partindo da responsabilidade de prevenir erros de todos os envolvidos no processo de trabalho.

Continuando na mesma vertente do entendimento frente a utilização do protocolo de cirurgia segura, encontrou-se relatos que afirmavam que o entendimento se baseava em medidas utilizadas para diminuir os eventos adventos nos procedimentos.

\section{"São medidas adotadas para reduzir o risco de eventos adversos, que podem acontecer durante o procedimento" (E12). \\ "O Protocolo é uma ferramenta utilizada para reduzir o número de incidentes relacionado aos procedimentos cirúrgicos ou qualquer outro procedimento realizado" (E6).}

O entendimento destes participantes parte do ponto de vista de que o protocolo é um procedimento primordial a ser seguido, pois evitará eventos adversos de baixo a média complexidade, que podem ocorrer durante os procedimentos cirúrgicos. A aceitação das ações de segurança do paciente, revela uma menor ocorrência de erros. Nessa concepção, os investigadores relacionam que cuidados simples podem minimizar erros e incidentes frequentes nos procedimentos realizados (SANTOS JS, et al., 2020).

Traçando um olhar a está compreensão, encontramos na fala dos enfermeiros a afirmação da importância da utilização do checklist, permitindo que os profissionais conheçam as informações pertinentes sobre o paciente de forma individual, para que não ocorram erros de identificação, tornando o serviço eficaz e transmitindo confiabilidade ao paciente.

Os enfermeiros têm melhores condições de reconhecer os riscos enfrentados pelos pacientes em centro cirúrgico, levando assim a integração da cultura da cirurgia segura (GUTIERRES LS, et al., 2019).

"Muito importante porque serve como garantia do processo de seguridade do paciente pela equipe, e evitando a morte desse paciente na mesa cirúrgica" (E17).

"É um processo muito importante para segurança do paciente onde irá ocorrer a redução de erros que levam a morte ou complicações aos pacientes submetidos a cirurgias" (E14).

As falas traduzem a valiosa importância de garantir a segurança dos pacientes, que será submetido a procedimentos cirúrgico pois, o checklist, pois esse é o segundo desafio Global para a Segurança do Paciente que nos apresenta o objetivo de promover a melhoria da segurança cirúrgica e reduzir as mortes e complicações durante a cirurgia (OMS, 2009)

$\mathrm{Na}$ busca pela qualidade da segurança do paciente, o enfermeiro é um profissional que pode traçar um processo de melhoria contínua do nível de cuidado com base no planejamento estratégico e nas orientações das boas práticas de enfermagem para reduzir os erros dos diferentes membros da equipe. Devido à proximidade com os pacientes e à atuação desses profissionais (GUTIERRES LS, et al., 2018).

\section{Importância do enfermeiro frente ao processo de cirurgia segura e no uso do checklist}

Cavalcante AKCB, et al. (2015) cita que, os profissionais de saúde, em especial os enfermeiros, são responsáveis por prestar assistência ao paciente de qualidade, eficiente e segura. Encontrou-se emergido dentro das falas dos participantes, o que pensam em relação à sua importância frente à segurança do paciente, e encontrou-se nos achados os que acreditam ser de sua responsabilidade a aplicação e conferência do checklist, pois são capazes de detectar possíveis erros e preveni-los. 
"É muito importante, é um momento em que pode se decidir ou não a realização de uma cirurgia, pois se não estiver atendendo aos critérios do checklist, o paciente não estará apto a realizar a intervenção e diante disse prevenirei as complicações" (E1).

"É fundamental para a supervisão do serviço, e dizer se o paciente está apto ou não a realizar a cirurgia, além de ser mediado da humanização do processo evitando a ocorrências de eventos adversos, pois o paciente não é um mero objeto que necessita de intervenção, estamos tratando pessoas" (E16).

O checklist é um instrumento eficaz para avaliação se o paciente está apto ou não a realizar o procedimento cirúrgico e caso ocorra alguma intercorrência o enfermeiro é capaz de intervir e solucionar, além de poder agir de forma humanizada.

Firmando os achados das falas Vazatta CAS (2019) nos coloca que, a utilização do checklist contribui para o trabalho seguro, uma vez que quando o profissional avalia de forma verídica maiores informações serão obtidas e registradas no instrumento, reduzindo as chances de falhas e de eventos adversos.

Algumas falas apontam que o processo de cirurgia segura deveria iniciar do enfermeiro, pois é o que acompanha rotineiramente o paciente e assim saberia direcionar toda a equipe a se dirigir ao paciente correto.

"O enfermeiro e o responsável inicial para o processo de cirurgia segura, pois como passo o dia acompanhando o paciente, e conheço seu quadro clinico conseguirei direcionar o paciente certo para a equipe é assim evitaria erros de troca de pacientes" (E12).

O profissional enfermeiro desenvolve o seu papel como gestor e pode possuir diversas atribuições nesta função dentre elas a de supervisão e assistencial, onde deverá atuar no auxílio da correta execução dos protocolos cirúrgicos e principalmente na prevenção de erros durante o procedimento no setor, inclusive na troca de pacientes por conta de nomes parecidos (VASCONCELOS MGV, et al., 2018).

Trazendo o olhar quanto a função do enfermeiro precursor da comunicação efetiva entre a equipe encontrou-se nas falas trechos que exemplificam essa afirmação:

"O enfermeiro proporciona uma comunicação entre a equipe multiprofissional, pois ele traz o checklist preenchido com as informações sobre a realidade do paciente se o mesmo poderá executar o procedimento destinado" (E6).

"Muito importante pois o enfermeiro tráz o fortalecimento da comunicação multidisciplinar e essa comunicação acarreta a prevenção de eventos adversos no paciente" (E7).

O enfermeiro é o responsável por levar as informações referente ao paciente após a realização do checklist e assim comunicar a equipe quanto sua situação, essa comunicação proporciona o fortalecimento da equipe e assim redução dos eventos adversos por falta de informações.

Segundo Oliveira AHS, et al. (2020), o checklist e considerado um instrumento para assistência, oportunizando a união da equipe e diminuindo as complicações operatórias. Contudo o método de aplicação do checklist cirúrgico mesmo que aparentemente simples, do ponto de vista administrativo, é considerado um sistema complexo, por conta da resistência dos profissionais a transformações.

A comunicação torna-se imprescindível, pois possibilita ao enfermeiro se aproximar de sua equipe e dos demais profissionais compreender as atividades realizadas, compartilhar ideias e visões e criar interdependência para o desenvolvimento do trabalho por meio da equipe (SANTOS MC e BERNARDES A, 2010). Silva LARS, et al. (2019) aponta que além da comunicação ainda existe o planejamento da assistência por meio da aplicação da Sistematização de Assistência de Enfermagem (SAE), permitindo ao enfermeiro realizar diagnósticos e planejar as ações de enfermagem, acompanhar e avaliar a evolução do paciente. 
A sistematização da assistência de enfermagem perioperatória (SAEP) é uma ferramenta valiosa e eficaz, utilizado por toda a equipe de enfermagem, criado para minimizarmos riscos e complicações durante o período perioperatório, para prestarmos uma assistência segura, continua e humanizada (MENDES PJA, et al., 2019).

"Evitar erros com paciente errado, cirurgia errado, reações alérgicas etc... O enfermeiro passa a conhecer as possíveis complicações cirúrgicas, aquelas inevitáveis" (E13).

"Conseguimos evitar inúmeros erros, o mais recorrente e trazer para a mesa cirúrgica o paciente errado, ou durante o procedimento cirúrgico descobrir que o membro que está sendo operado infelizmente não é o danificado" (E8).

A responsabilidade de gerencia todo o fluxo para o procedimento cirúrgico recai como competência primordial do enfermeiro, mais a funcionalidade de todo o desenrolar até a cirurgia envolve uma equipe que necessita está atenta.

\footnotetext{
"Pacientes errado, devido à falta de realização do check é identificação correta do paciente" (E5).
}

"Erros durante a demarcação do sitio cirúrgico, a pressa em finalizar o procedimento devido à demanda de atendimento ocasionar a erros gravíssimos" (E11).

Os eventos adversos como citados nas falas são mais recorrentes que se imagina, então o check listse torna peça fundamental para minimizar tais erros, prováveis erros são produzidos em consequência aos eventos adversos (SILVA HR, et al.,2019).

\section{Dificuldades para realização do checklist no serviço}

Segundo Cardoso TIC, et al. (2020), um dos desafios achados para implementação do checklist não é a deficiência de conhecimento sobre os protocolos, mas as dificuldades em preenchê-lo de modo correto, por causa da rapidez no processo e no encaminhamento do paciente. Também encontrou-se este achando nas falas dos participantes:

"Muitas vezes o período entre a entrada do paciente na sala vermelha e o encaminhamento deste ao CC é muito curto, dificultando a avaliação do mesmo. $A$ comunicação entre equipe médica e de enfermagem pode melhorar contribuindo para avaliação do checklist de cirurgia segura" (E15).

"Por hora esta corrido demais pois alguns médicos não avisam qual paciente vai para o CC, logo temos que responder o checklist muito rápido, SSVV fica incompleto devido falta de tempo. Precisa melhorar a comunicação previa do médico com a equipe de enfermagem, ou pelo menos um mapa cirúrgico com os nomes dos pré-operatórios" (E13).

O aumento das cirurgias em paciente em situação de emergência, a necessidade de intervenção de forma rápida é assim a realização do checklist fica fragilizada, parâmetros essenciais deixam de ser verificados, rotina agitada são umas das causas de dificuldades de implementa o checklist e assim desenvolve o protocolo de cirurgia segura (HENRIQUES AHB, et al., 2016).

Barreiras são detectadas em aplicar o checklist, em especial na diminuição da comunicação médico/enfermeiro, pois devido à sobrecarga de trabalho os médicos buscam agilizar seu lado da assistência sem se preocupar com o lado da equipe de enfermagem (BEORDO JR,2021). Os participantes confirmam estas informações nas falas a seguir:

"Dificuldade na comunicação e resistência de profissionais mais antigos, pressa em executar a ação deixando a avaliação pré-anestésica e pré-operatório de lado" (E7). 
"A equipe médica só se preocupa em realizar a sua assistência sem se importar com a relevância da equipe de enfermagem nesse processo" (E03).

$O$ processo precisa ter continuidade de forma que todos os profissionais que compõem a equipe desempenhem o seu papel mediando comunicação, sem que haja falhas, e os médicos saibam a relevância dos cuidados que são essências que partem da competência da equipe de enfermagem.

O enfermeiro tem parte essencial na execução do checklist, com o preparo do paciente no pré-operatório até a sua saída da sala de cirurgia, colaborando na minimização dos erros na entrada na sala operatória, durante e até sua saída do hospital, sendo necessário o respeito de todas estas etapas (ROCHA RG, et al., 2021). Outro achado relevante nas falas foi a resistência na adesão do checklist devido à sobre carga de serviço:

"Já temos muito serviço para fazer, a sobre carga de serviço é muito grande isso vai só dificultar o processo, melhor deixar como está mesmo" (E18).

A sobrecarga de trabalho é um dos fatores mais recorrentes para resistência na adesão de protocolos, conforme notado na fala. O enfermeiro, especialmente o que atua em âmbito hospitalar, está exposto a situações que ocasionam efeitos danosos à saúde, decorrentes da própria organização do trabalho, trazendo a resistência em aceitar novas competências e práticas, como a aplicação do checklist de forma rotineira (MUNIZ DC, et al., 2019).

Também mostra-se a necessidade do acrescentamento de enfermeiros em conseqüência da sobrecarga de atendimento no centro cirúrgico, com a finalidade de acompanhar o paciente de forma integra com qualidade (PANZETTI MN, et al., 2020).

\section{Quarta etapa: hipótese de solução}

Nesta etapa contamos com a participação do Núcleo de Segurança do Paciente (NSP) do hospital, no qual ele foi um colaborador importante para a discussão do confronto dos dados encontrados nas falas dos participantes com a realidade vivenciada pelos mesmos. Tal fato se deve a característica que este núcleo tem Planos de Segurança ao paciente em desenvolver estratégia para transformar o cenário hospitalar.

As hipóteses desenvolvidas a respeito das possíveis soluções para o problema: Promover a troca de conhecimento e experiências entre os profissionais enfermeiros acerca da rotina de uso do checklist, com o objetivo de aperfeiçoar os processos de trabalho dentro do protocolo de cirurgia segura. Estimular a participação do profissional no processo de elaboração e de implantação do checklist. Promover, através de encontros (roda de conversa, oficinas), um resgate das informações e reflexões, sobre os problemas que dificultam o uso do checklist de cirurgia segura. Enfatizar a importância do preenchimento adequado do checklist, pois tais informações são fundamentais no que tange a segurança do paciente.

\section{Quinta etapa: aplicação à realidade}

Foi proposto a sensibilização do uso do protocolo de cirurgia segura, para os enfermeiros do pronto socorro, no intuito de viabilizar a assistência dos pacientes que iriam para o centro cirúrgico e assim reduzir e/ou minimizar os erros durante os procedimentos cirúrgicos.

Como intervenção foi realizado rodas de conversa com os profissionais enfermeiros, promovendo assim uma discussão crítica e reflexiva sobre o processo de trabalho envolvendo toda a caminhada na cirurgia segura para o paciente. As rodas de conversa foram agendadas em 5 dias, no período manhã e noite, com o tempo médio de duração de cada sessão de duas horas e trinta minutos. Explicamos os aspectos e conceitos fundamentais sobre o protocolo de cirurgia segura, mostrando como realizar o preenchimento correto do checklist.

Foi disposto uma caixa com as perguntas de todos os procedimentos que estão no cheklist, cada participante pegava um papel lia em voz alta, após a leitura perguntávamos qual era importância daquele procedimento que estava descrito no papel e em que momento dentro do preenchimento do checklist, pertencia aquele procedimento. Como por exemplo: qual importância de preenche o termo de 
consentimento? Em que momento se realizar está ação? Após responder o participante, se dirigia a placa e colocava em qual momento é o mais recomendado para cada situação. Diante disso, foi possível ouvir os anseios, questionamentos e dúvidas quanto à utilização do checklist, foi o momento que os participantes conseguiram transmitir aquilo que os inquietava em relação a temática, ocorrendo uma reflexão, e a sensibilização dos profissionais envolvidos na ação.

\section{CONCLUSÃO}

Este estudo possibilitou a reflexão e conscientização sobre o tema apresentado, percebemos ao finalizar a educação permanente, que os participantes apresentaram harmonia no preenchimento correto da LVCS, e a conscientização em se envolver no processo, através da roda de conversa entramos em debates e discussão de diferente concepção. Desta forma consequentemente propiciou aperfeiçoamento na qualidade de assistência visando a segurança do paciente, do mesmo modo a consolidação entre a equipe multiprofissional.

\section{REFERÊNCIAS}

1. BARBOSAGA, et al. A percepção dos profissionais de enfermagem do centro cirúrgico em relação aos benefícios da implantação do protocolo de cirurgia segura em um hospital filantrópico no município de Sete Lagoas, MG. Revista Brasileira de Ciências da Vida, 2018; 6(3).

2. BORGES F, et al. Grau da cultura de segurança do paciente na percepção da equipe multiprofissional hospitalar. Varia Scientia-Ciências da Saúde, 2016; 2(1): 55-66.

3. BEORDOJR. Vista do Segurança do paciente por meio da aplicação adequada do checklist de cirurgia segura. Global Academic Nursing Journal, 2021; 2(1): e88.

4. CARDOSO TIC, et al. Percepções dos profissionais de enfermagem na aplicação do checklist de cirurgia segura. Journal of Nursing and Health, 2020; 10(1): e20101010.

5. CAVALCANTE AKCB, et al. Cuidado seguro ao paciente: contribuições da enfermagem. Revista Cubana de Enfermería, 2015; 31(4): 1561-2961.

6. CRIADO JVN, et al. Processo de implementação do protocolo de cirurgia segura. Revista Cubana de Enfermería, 2017; 33(1): 1561-2961.

7. GUTIERRES LS, et al. Boas práticas para segurança do paciente em centro cirúrgico: recomendações de enfermeiros. Rev. Bras. Enferm, 2018; 71(6): 2775-2782

8. GUTIERRES LS, et al. "Práticas de enfermeiros na gestão do cuidado de enfermagem para a promoção da segurança do paciente no centro cirúrgico", presente do Universidade Federal de Santa Catarina, Florianópolis, SC, Brazil. Revista Latino-Americana de Enfermagem [online], 2019; 27: 3108.

9. HENRIQUES AHB, et al. Assistência de enfermagem na segurança do paciente cirúrgico: revisão integrative. Cogitare Enferm., 2016; 21(4): 01-09.

10. LOPES MCR, et al. Atuação da enfermagem no processo de cirurgia segura. ReTEP [Internet] 2018; 10(4): 34-39.

11. MENDES PJA, et al. Atuação do enfermeiro na prevenção de eventos adversos no centro cirúrgico, utilizando saep. Editorial Bius, 2020; 19(13).

12. MUNIZ DC, et al. A saúde do enfermeiro com a sobrecarga de trabalho. Revista de Iniciação Científica e Extensão, 2019; 2(2): 274-279,

13. MINAYO MCS. O desafio do conhecimento: pesquisa qualitativa em saúde (13르 Ed). Editora Hucitec., 2013.

14. OLIVEIRAAHS, et al. Implementação do checklist de cirurgia segura: desafios e o papel da enfermagem. Única Cadernos Acadêmicos, 2020; 1, 1(6).

15. OLIVEIRA MCB, et al. Adesão do cheklist cirúrgico à luz da Cultura de segurança do paciente. Revista SOBECC, 2018; 23(1): 36-42.

16. OMS, ORGANIZAÇÃO MUNDIAL DA SAÚDE. Segundo desafio global para a segurança do paciente: Cirurgias seguras salvam vidas (orientações para cirurgia segura da OMS). Ministério da Saúde; Agencia Nacional de

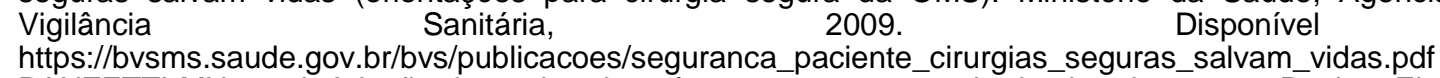

17. PANZETTI MN, et al. Adesão da equipe de enfermagem ao protocolo de cirurgia segura. Revista Eletrônica Acervo Saúde, 2020; 12(2): 25-19

18. ROCHA RG, et al. Boas práticas na utilização do checklist de cirurgia segura por enfermeiros no período transoperatório. Glob Acad Nurs[Internet]., 2021;2(1): 86.

19. SANTOS JS, et al. Test check pilot list of safe surgery: experience report. Rev Enferm UFPI, 2020; 6(1).

20. SANTOS MC, BERNARDES A. Comunicação da equipe de enfermagem e a relação com a gerência nas instituições de saúde. Revista Gaúcha de Enfermagem [online]. 2010; 31(2): 359-366.

21. SILVA ACA, et al. A segurança do paciente em âmbito hospitalar: Revisão integrativa da literatura. Cogitare Enfermagem, 2016; 21(5): 2176-9133.

22. SILVA HR, et al. Percepção da equipe de enfermagem quanto as contribuições da utilização do checklist de cirurgia segura. Revista Enfermagem Atual In Derme, 2019; 87(25).

23. SILVA LARS, et al. $O$ arco de maguerez como metodologia ativa na formação continuada em saúde. EDUCAÇÃO, 2020; 8(3): 41-54.

24. VAZATT CAS. Percepção dos profissionais da enfermagem frente a utilização do checklist de cirurgia segura. URI Erechim, 2019. 